\title{
Tunable Networks from Thiolene Chemistry for Lithium Ion Conduction
}

\author{
Catherine N. Walker, ${ }^{\dagger}$ Craig Versek, ${ }^{\ddagger}$ Mark Touminen, ${ }^{\ddagger}$ and Gregory N. Tew ${ }^{*} \dagger$ \\ ${ }^{\dagger}$ Department of Polymer Science and Engineering, University of Massachusetts, Amherst, Massachusetts 01003, United States \\ ${ }^{\ddagger}$ Department of Physics, University of Massachusetts, Amherst, Massachusetts 01003, United States
}

\section{Supporting Information}

ABSTRACT: End-functionalized poly(ethylene glycol) (PEG) and polydimethylsiloxane (PDMS) were cross-linked by a thiolene reaction with a tetra-functional thiol to create robust, tunable networks. These networks were loaded with increasing amounts of lithium bis(trifluoromethane sulfonyl imide) (LiTFSI), and their ion conductivity was measured. A wide range of salt loading was achieved, allowing the investigation of both salt-in-polymer and polymer-in-salt regimes. Thermal, mechanical, and ion conductivity properties of LiTFSI-loaded PEG and PEG-PDMS networks were measured. Even at high salt loadings, both networks maintained rubber-like characteristics, which were stable over a range of temperatures $\left(30-90{ }^{\circ} \mathrm{C}\right)$. The PEG network with the highest salt loading showed the greatest ion conductivity, $6.7 \times 10^{-4} \mathrm{~S} \mathrm{~cm}^{-1}$ at $30{ }^{\circ} \mathrm{C}$, as measured by impedance spectroscopy. This system provides a route to optimize lithium ion conduction and mechanical properties.

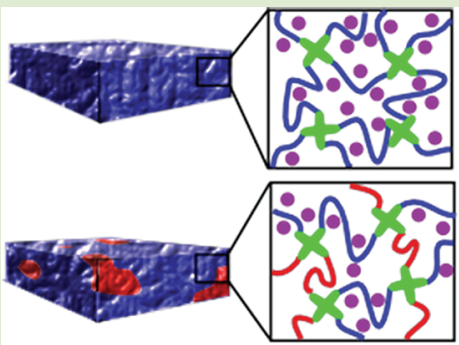

N PEG

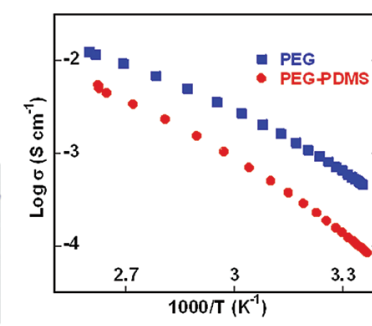

4SH

LiTFSI
A ccess to affordable, clean energy is a well-recognized scientific and technical challenge of this century. ${ }^{1}$ Although many different opportunities are being investigated, every solution involves better batteries, as they represent the main energy storage device. ${ }^{2}$ Despite widespread use, current batteries are far from their theoretical storage potential, and significant improvements can be made (up to $95 \%$ of theoretical values) by eliminating components such as the separator. ${ }^{3}$ Among commercially available batteries, lithium ion batteries are attractive because they have the highest energy density. ${ }^{4,5}$ One important area of research focuses on replacing the current liquid or gel electrolyte of these batteries with a solid material. This is expected to double the energy density and eliminate the volatile organic solvents, which pose a fire hazard. ${ }^{4,6}$ For a solid material to replace liquid and gel electrolytes, it should have a minimum ion conductivity of $10^{-3}$ $S \mathrm{~cm}^{-1}$ throughout the expected range of operating temperatures. $^{2,4}$ At the same time, solid electrolytes should have enough mechanical integrity to replace separators currently used in the liquid electrolyte design. While many material systems have been examined, ${ }^{4-7}$ few exhibit the necessary conductivity or mechanical properties; simultaneously improving both remains a challenge. Soft materials enable rapid chainrelaxation and thus higher conductivity, while stiffer materials, which yield more appropriate mechanical properties, generally have a lower conductivity. ${ }^{8}$ A notable exception is the poly(ethylene glycol) (PEG)-polystyrene block copolymer system reported by Balsara and co-workers that showed ion conducting properties decoupled from the mechanical properties without a large decrease in ion conductivity, yielding materials with a high shear modulus, $10-100 \mathrm{MPa}$, and ion conductivity of $3.6 \times 10^{-4} \mathrm{~S} \mathrm{~cm}^{-1}$ at $90{ }^{\circ} \mathrm{C}^{9}$.

Since Wright discovered the ion conducting properties of PEG, ${ }^{10}$ this material has been extensively studied as the main component of solid electrolytes and remains one of the best lithium ion conductors known. However, PEG conducts lithium ions through chain relaxation, which typically creates a trade-off between robust mechanical properties and high ion conductivity. ${ }^{7,11}$ In addition, the crystallinity of PEG reduces its ion conductivity. Efforts to limit this crystallization have included numerous branch polymers with reported conductivities of $7 \times 10^{-5}$ to $10^{-3} \mathrm{~S} \mathrm{~cm}^{-1}$ at $90{ }^{\circ} \mathrm{C} .{ }^{12,13}$ Similarly, crosslinking can reduce crystallinity while increasing mechanical stability, ${ }^{2}$ giving materials with moduli on the order of $10^{4}-10^{6}$ $\mathrm{Pa}$ and ion conductivity on the order of $10^{-5} \mathrm{~S} \mathrm{~cm}^{-1}$ at 25 ${ }^{\circ} \mathrm{C} .{ }^{14-16}$ However, the cross-linking must be efficient since loops, dangling ends, or other defects compromise the mechanical properties. ${ }^{5}$ It has also been shown that materials with low $T_{\mathrm{g}}$ generally have better ion conductivities. ${ }^{17-19}$ This leads to the study of PDMS-based materials including comblike polymers consisting of a polymethylhydrosiloxane (PMHS) backbone with oligo(ethylene glycol) side chains. These polymers alone did not have sufficient mechanical properties to yield free-standing films so they were entrapped in crosslinked PEG-dimethacrylate. The resulting semi-interpenetrating

Received: February 23, 2012

Accepted: May 21, 2012 


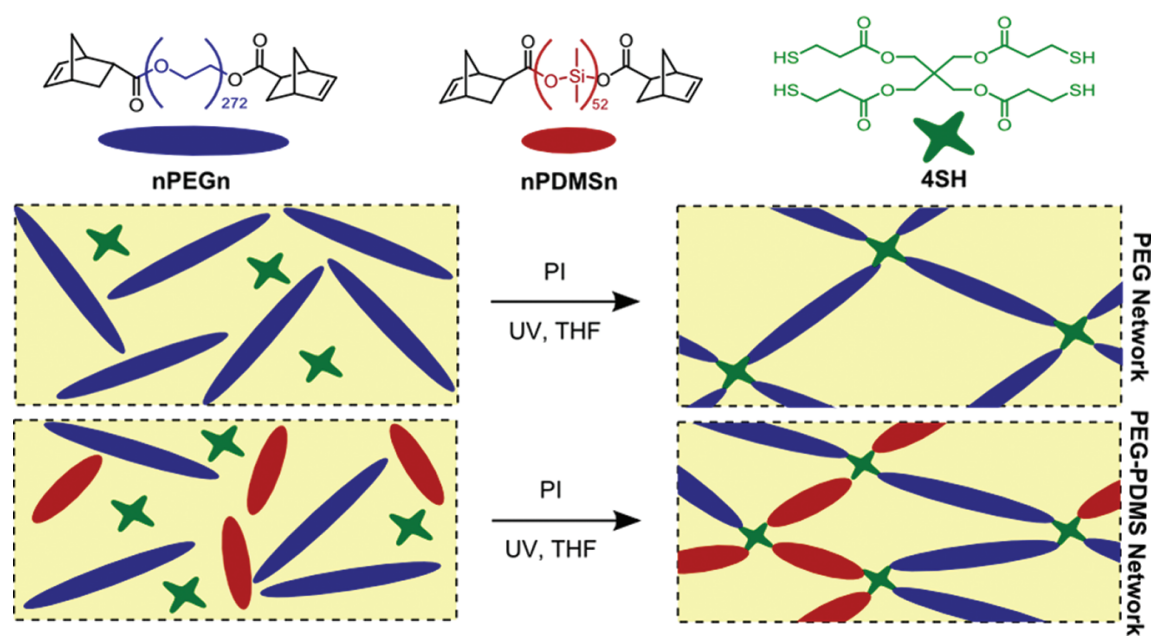

Figure 1. End-functionalized precursors and synthetic conditions for the formation of PEG and PEG-PDMS cross-linked networks. The structure of nPDMSn as been abbreviated for space and clarity; the full structure can be found in the SI.
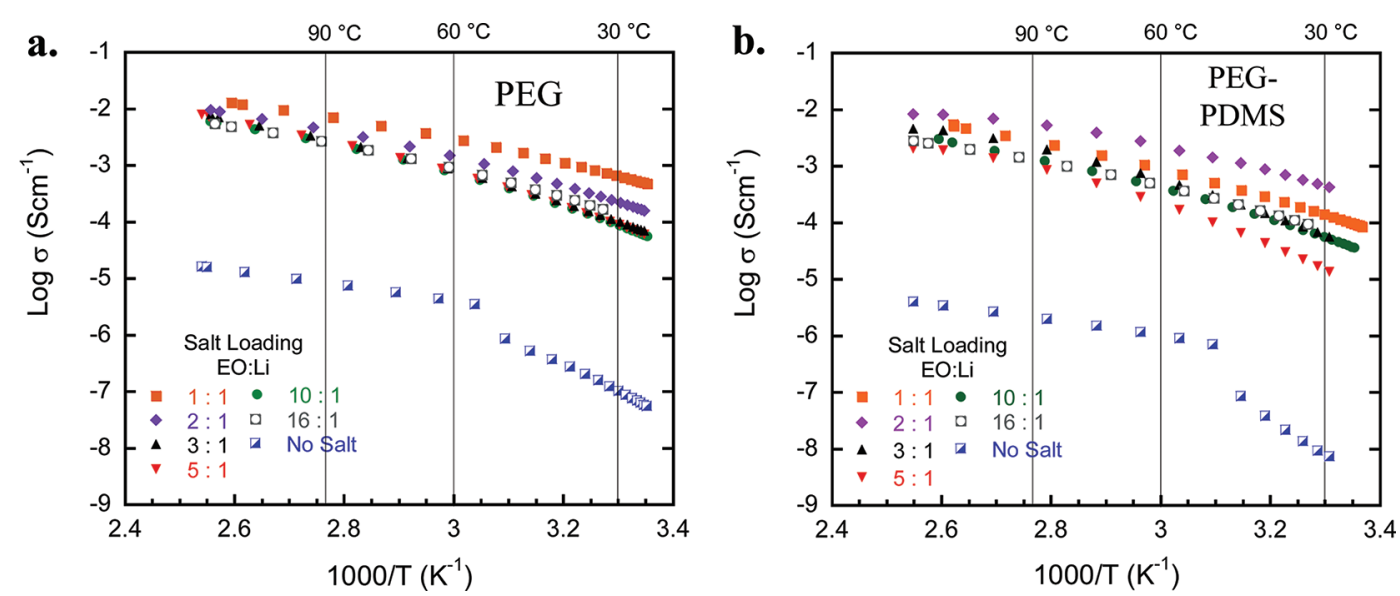

Figure 2. Ion conductivity of (a) PEG networks and (b) PEG-PDMS networks in Arrhenius plots. Approximate temperatures in ${ }^{\circ} \mathrm{C}$ are given as a reference at the top of the plot. The salt loadings, $\mathrm{EO} / \mathrm{Li}$, are given in the legend.

networks were free-standing and exhibited an ion conductivity of approximately $10^{-4} \mathrm{~S} \mathrm{~cm}^{-1}$ at $37^{\circ} \mathrm{C}$. ${ }^{17}$

The amount of salt dissolved in the polymer electrolyte also affects ion conductivity. ${ }^{4}$ Generally, PEG-based polymer electrolytes exhibit a maximum conductivity at fairly low salt loadings such as a molar ratio of ether oxygens to lithium ions $(\mathrm{EO} / \mathrm{Li})$ of 16 . This trend is due to the lithium ions acting as additional physical cross-links between polymer chains, increasing the $T_{\mathrm{g}}$ and reducing the molecular mobility. ${ }^{9,20,21}$ At significantly higher salt loading, the polymer $T_{\mathrm{g}}$ decreases, causing conductivity to increase. This regime has been dubbed "polymer-in-salt" as opposed to the conventional "salt-inpolymer" example described above. ${ }^{20}$ Provided the molecular weight of the linear, noncross-linked polymer is high $\left(>8 \times 10^{6}\right.$ $\left.\mathrm{g} \mathrm{mol}^{-1}\right)$, it takes as little as $10 \mathrm{wt} \%$ to give the material rubbery properties (shear modulus of $10^{4} \mathrm{~Pa}$ ) despite the high salt content. ${ }^{22}$ Because networks have an infinite molecular weight, they should also be able to exhibit rubbery properties at high salt loading, for example $\mathrm{EO} / \mathrm{Li}=1$.

In this report, hydroxyl-terminated, narrow polydispersity polymers were quantitatively end-functionalized with reactive norbornene end groups by the highly efficient Mitsunobu reaction. Figure 1 shows the cross-linking chemistry used to synthesize the two types of networks investigated in this study.
The telechelic polymer precursors were cross-linked by a tetrafunctional thiol in a photoinitiated thiolene reaction to create a well-defined network. This chemistry is an attractive method for solid electrolyte preparation because it is known to be highly efficient, yield networks with few defects ${ }^{23,24}$ and can accommodate a wide variety of precursor polymers. In the PEG-PDMS networks, the molar ratio of PEG to PDMS is $1: 1$. As the PDMS degree of polymerization is much less than the PEG, their volume fractions are approximately 0.3 and 0.7 , respectively, before salt loading. Once the networks were formed, the gels were dried thoroughly and weighed. They were then swollen in tetrahydrofuran (THF) solutions of varying lithium bis(trifluoromethanesulfonyl imide) (LiTFSI) concentrations. After swelling for three days, they were thoroughly dried and weighed again. The difference in weight was used to calculate the molar ratio of ether oxygens in the PEG chains to lithium ions $(\mathrm{EO} / \mathrm{Li})$. (For further details on the network preparation and salt loading, see the experimental section in the Supporting Information (SI).)

The ion conductivity of PEG and PEG-PDMS networks obtained from impedance spectroscopy is shown in Figure 2. Loading either network with lithium salt increases the ion conductivity by at least 2 orders of magnitude when compared to the control networks which contain no added salt. The PEG 

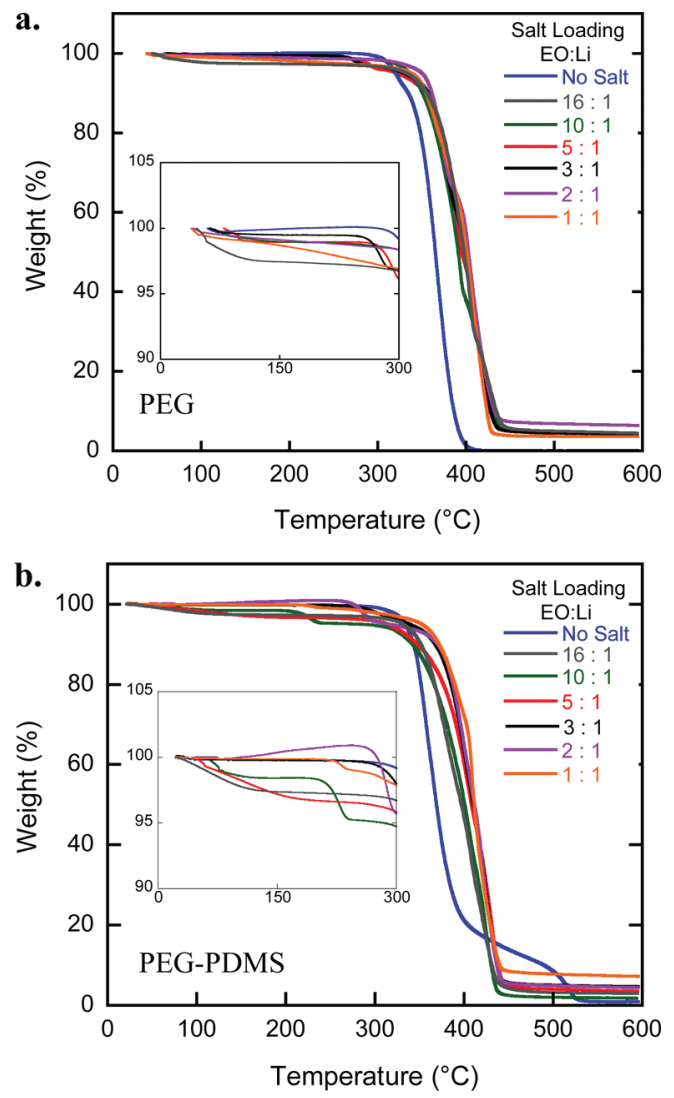

Figure 3. TGA of (a) PEG networks and (b) PEG-PDMS networks with a heating rate of $10{ }^{\circ} \mathrm{C} / \mathrm{min}$. Figure insets show only slight weight loss due to solvent or moisture.

and PEG-PDMS networks show an ion conductivity $(8.6 \times$ $10^{-5}$ to $6.7 \times 10^{-4}$ and $1.3 \times 10^{-5}$ to $4.2 \times 10^{-4} \mathrm{~S} \mathrm{~cm}^{-1}$, at 30 ${ }^{\circ} \mathrm{C}$, respectively $)$ similar to other materials in the field, $(1 \times$ $10^{-5}$ to $\left.6 \times 10^{-4} \mathrm{~S} \mathrm{~cm}^{-1}\right) \cdot{ }^{13-19}$ The conductivity as a function of temperature shows non-Arrhenius behavior with a decreasing slope at lower temperatures. The control networks show a different behavior, which includes a sharp decrease in conductivity at approximately $55{ }^{\circ} \mathrm{C}$ due to the crystallization of the PEG chains. In both networks, the highest salt loadings, $\mathrm{EO} / \mathrm{Li}=1: 1$ and $2: 1$, show a slight increase in conduction over conventional salt loadings, $\mathrm{EO} / \mathrm{Li}=10: 1$ and $16: 1$. As expected, ion conductivity in the PEG-PDMS networks is slightly lower than that of the PEG networks due to the reduced volume fraction of PEG.

The values reported in Figure 2 are taken from a cooling curve after holding the samples at $120{ }^{\circ} \mathrm{C}$ under vacuum for several hours. It is well-known that PEG and LiTFSI are hygroscopic. This "baking" process allows for the removal of any residual solvents or moisture from the salt loaded networks. The ion conductivity during this baking process shows that all networks reach a stable state before the samples are cooled, as seen in Figure S2 of the SI where the ion conductivity is plotted against time. It has been demonstrated that the effect of moisture on conductivity is largely reversible once the water is removed with heat. ${ }^{25}$ It is logical that the effects of any other plasticizing solvents are also largely reversible. Based on this assumption, THF was chosen as the solvent for salt loading because it is easy to remove on heating.

To corroborate the effectiveness of the baking process, thermogravimetric analysis (TGA) measurements were per-
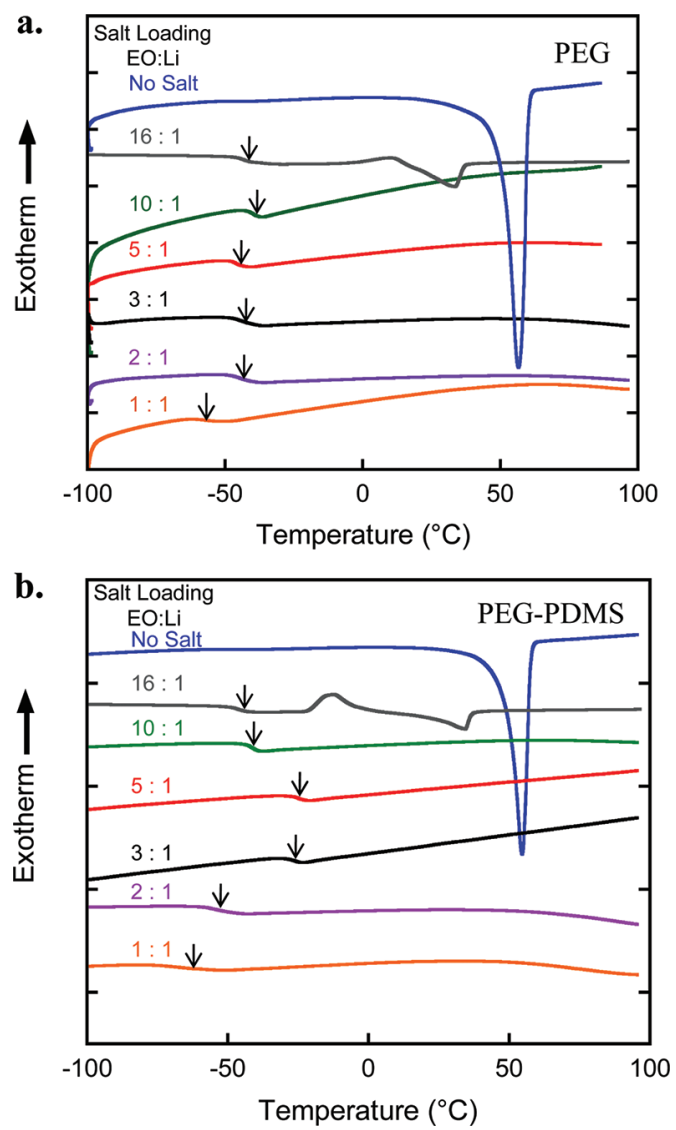

Figure 4. DSC traces of (a) PEG and (b) PEG-PDMS networks. All traces are taken from the second heating scan, with a scanning rate of $10{ }^{\circ} \mathrm{C} / \mathrm{min}$. Data are offset on the $Y$-axis for clarity. $T_{\mathrm{g}}$ of PEG is marked with arrows.

formed. As shown in Figure S3 of the SI, the pure salt, LiTFSI, contains $20 \%$ weight moisture which is removed upon heating to $90{ }^{\circ} \mathrm{C}$, and very little change is observed $(<5 \mathrm{wt} \%)$ between $90{ }^{\circ} \mathrm{C}$ and the decomposition temperature of $400{ }^{\circ} \mathrm{C}$. Plots of weight loss as a function of temperature for the salt-loaded networks (Figure 3) show that the samples are quite stable up to $320{ }^{\circ} \mathrm{C}$ which allows for a wide range of operating temperatures. This data also confirms that the "baking" process eliminates the majority of the moisture observed in the TGA curve for the pure salt with less than $5 \mathrm{wt} \%$ loss for any sample up to $320{ }^{\circ} \mathrm{C}$ (Figure 3 insets). The sample with the greatest weight loss (5\%) below $320{ }^{\circ} \mathrm{C}$, PEG-PDMS EO/Li $=10: 1$, shows a conductivity similar to all of the other samples indicating that the weight loss, possibly related to moisture, above $180{ }^{\circ} \mathrm{C}$ does not enhance conductivity compared to the other networks.

LiTFSI is known for its ability to disrupt crystallinity due to the irregular shape of the TFSI anion, making this salt a popular choice for PEG-based ion conducting materials. ${ }^{26}$ Differential scanning calorimetry (DSC) measurements (Figure 4) indicate that any salt loading greater than $\mathrm{EO} / \mathrm{Li}=10: 1$ completely disrupts the crystallinity of the PEG chains for both sets of networks. The crystallinity in both the PEG and the PEGPDMS networks for $\mathrm{EO} / \mathrm{Li}=16: 1$ is much less than the crystallinity in the control networks that do not contain salt. However, none of the salt-containing samples show a crystallization-related decrease in conductivity in the temperature range investigated. 

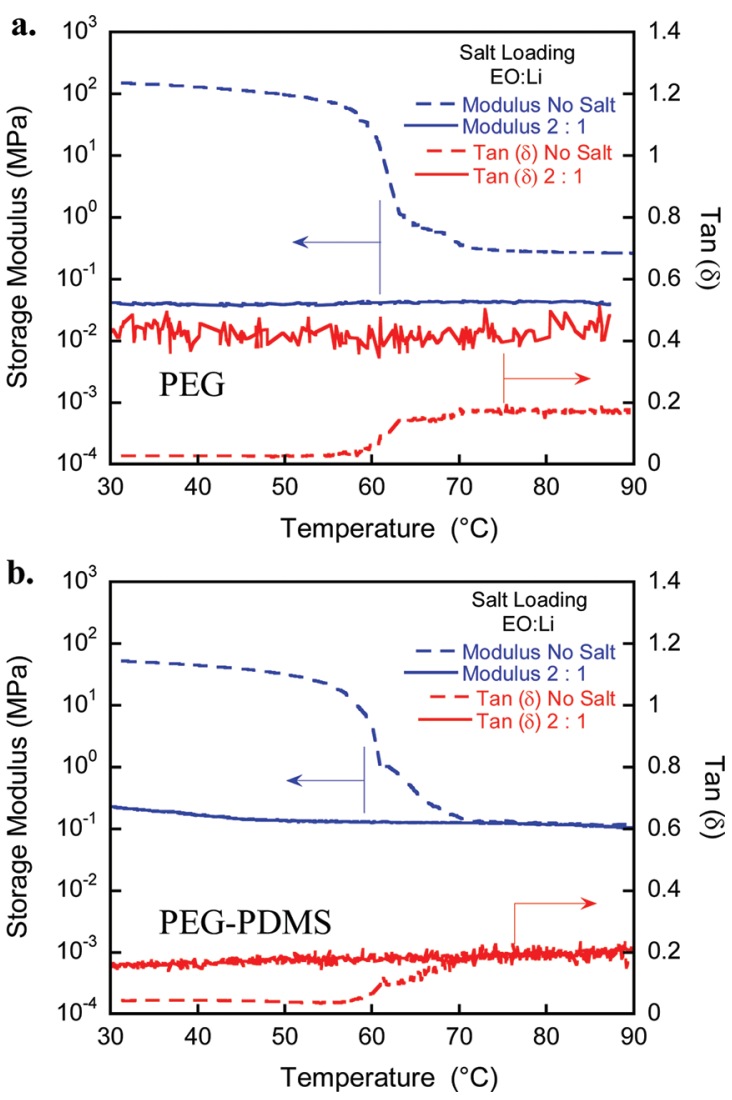

Figure 5. Storage modulus and $\tan (\delta)$ of (a) PEG networks and (b) PEG-PDMS networks measured at $1 \mathrm{~Hz}$ as a function of temperature. The data for the control sample in both network are given by dashed lines while the $\mathrm{EO} / \mathrm{Li}=2$ are solid. The modulus values are shown in blue, while the $\tan (\delta)$ values are shown in red.

All of the salt-loaded networks show a $T_{\mathrm{g}}$ for the PEG component between $-65{ }^{\circ} \mathrm{C}$ and $-20{ }^{\circ} \mathrm{C}$. In the PEG-PDMS networks, $T_{\mathrm{g}}$ goes through a maximum at $\mathrm{EO} / \mathrm{Li}=5: 1$ and then decreases as the salt loading continues to increase. This sample also had the lowest conductivity values. Although it is less pronounced, the $T_{\mathrm{g}}$ drops at the highest salt loading in the PEG networks. To illustrate this trend more clearly, $T_{\mathrm{g}}$ versus salt loading is plotted in Figure S4 of the SI. This trend indicates that the range of salt loading includes both the salt-in-polymer and the polymer-in-salt regimes. At low salt loadings, the $T_{\mathrm{g}}$ increases as salt loading increases possibly because the lithium cations serve as additional physical cross-links between chains, decreasing the molecular mobility of the polymer chains. ${ }^{21}$ However, once the salt is the major component in the system, the $T_{\mathrm{g}}$ decreases similar to previous observations. ${ }^{20,22}$ This regime change occurs when the PEG chains are saturated and cannot complex any additional cations. ${ }^{22}$

Dynamic mechanical analysis (Figure 5) shows that both PEG and PEG-PDMS salt-loaded networks have stable mechanical properties in the temperature range probed. As expected, both salt-free control samples show a large drop in storage modulus and a slight increase in $\tan (\delta)$ as the PEG crystals melt at approximately $60{ }^{\circ} \mathrm{C}$. Even with PEG in the crystalline state, the effect of the soft PDMS chains can be seen as the storage modulus of the control PEG-PDMS network (50 $\mathrm{MPa}$ ) is lower than that of the PEG control network (150 $\mathrm{MPa})$. The salt-loaded networks do not show this transition because they are predominately amorphous, corroborating the
DSC results. Instead, they maintain a relatively constant modulus over the temperature range investigated, $40 \mathrm{kPa}$ for PEG and $200 \mathrm{kPa}$ for PEG-PDMS. As with conductivity, these materials have comparable mechanical properties (approximately $1 \mathrm{MPa}$ ) to other materials in this field. ${ }^{13-16,27}$ Even at high salt loadings, both the PEG and the PEG-PDMS networks remain elastic, maintaining a rubbery-like modulus and $\tan (\delta)$ values much less than unity.

Here, we presented a chemical platform by which the mechanical properties and ion conductivity can be tuned to produce more effective materials. The mechanical properties can be modified by the incorporation of a nonion conducting component without greatly reducing the ion conductivity. As previously demonstrated in block-copolymer systems, ${ }^{9}$ the ion conductivity of the material is dependent on the volume fraction of PEG. We envisage that, using the same chemical platform, the mechanical properties can be further improved by substituting PDMS with a stiffer nonion conducting component, while maintaining the same ion conducting properties demonstrated in the PEG-PDMS networks. The ion conduction and mechanical properties could also be tuned by changing the molecular weight of the precursor polymers or the volume fraction of the network components.

\section{ASSOCIATED CONTENT}

\section{S Supporting Information}

Experimental, conductivity versus time, and TGA of LiTFSI. This material is available free of charge via the Internet at http://pubs.acs.org.

\section{AUTHOR INFORMATION}

\section{Corresponding Author}

*Fax: +1-413-545-0082; e-mail: tew@mail.pse.umass.edu.

Notes

The authors declare no competing financial interest.

\section{ACKNOWLEDGMENTS}

This work was supported by the Army Research Office (W911NF-09-0373) and the National Science Foundation (DMR-0820506). This work utilized facilities supported in part by the National Science Foundation Materials Research Science and Engineering Center (DMR 0820506) and Center for Hierarchical Manufacturing (CMMI 0531171). The authors would like to thank Dr. Federica Sgolastra and Ms. Katie Gibney for assisting with manuscript preparation.

\section{REFERENCES}

(1) World Energy Outlook 2011; International Energy Agency: Paris, 2011.

(2) Goodenough, J. B.; Kim, Y. Chem. Mater. 2010, 22, 587.

(3) Voith, M. Chem. Eng. News 2010, 88, 27.

(4) Agrawal, R. C.; Pandey, G. P. J. Phys. D: Appl. Phys. 2008, 41, 223001.

(5) Tarascon, J. M.; Armand, M. Nature 2001, 414, 359.

(6) Quartarone, E.; Mustarelli, P. Chem. Soc. Rev. 2011, 40, 2525.

(7) Meyer, W. H. Adv. Mater. 1998, 10, 439.

(8) Gorecki, W.; Jeannin, M.; Belorizky, E.; Armand, M. J. Phys.: Condens. Matter 1995, 7, 6823.

(9) Singh, M.; Odusanya, O.; Wilmes, G. M.; Eitouni, H. B.; Gomez, E. D.; Patel, A. J.; Chen, V. L.; Park, M. J.; Fragouli, P.; Iatrou, H.; Hadjichristidis, N.; Cookson, D.; Balsara, N. P. Macromolecules 2007, $40,4578$.

(10) Wright, P. V. Br. Polym. J. 1975, 7, 319. 
(11) Müller-Plathe, F.; van Gunsteren, W. F. J. Chem. Phys. 1995, 103, 4745 .

(12) Hawker, C. J.; Chu, F.; Pomery, P. J.; Hill, D. J. T. Macromolecules 1996, 29, 3831.

(13) Nishimoto, A.; Watanabe, M.; Ikeda, Y.; Kohjiya, S. Electrochim. Acta 1998, 43, 1177.

(14) Kono, M.; Hayashi, E.; Watanabe, M. J. Electrochem. Soc. 1998, $145,1521$.

(15) Trapa, P. E.; Won, Y.-Y.; Mui, S. C.; Olivetti, E. A.; Huang, B.; Sadoway, D. R.; Mayes, A. M.; Dallek, S. J. Electrochem. Soc. 2005, 152, A1.

(16) Tigelaar, D. M.; Meador, M. A. B.; Kinder, J. D.; Bennett, W. R. Macromolecules 2006, 39, 120.

(17) Oh, B.; Vissers, D.; Zhang, Z.; West, R.; Tsukamoto, H.; Amine, K. J. Power Sources 2003, 119-121, 442.

(18) Rossi, N. A. A.; Zhang, Z.; Schneider, Y.; Morcom, K.; Lyons, L. J.; Wang, Q.; Amine, K.; West, R. Chem. Mater. 2006, 18, 1289.

(19) Zhang, Z.; Jin, J.; Bautista, F.; Lyons, L.; Shariatzadeh, N.; Sherlock, D.; Amine, K.; West, R. Solid State Ionics 2004, 170, 233.

(20) Angell, C. A.; Liu, C.; Sanchez, E. Nature 1993, 362, 137.

(21) Kakihana, M.; Schantz, S.; Torell, L. M. J. Chem. Phys. 1990, 92, 6271.

(22) Fan, J.; Angell, C. A. Electrochim. Acta 1995, 40, 2397.

(23) Hoyle, C. E.; Lee, T. Y.; Roper, T. J. Polym. Sci., Part A: Polym. Chem. 2004, 42, 5301.

(24) Fairbanks, B. D.; Schwartz, M. P.; Halevi, A. E.; Nuttelman, C. R.; Bowman, C. N.; Anseth, K. S. Adv. Mater. 2009, 21, 5005.

(25) Munshi, M. Z. A.; Owens, B. B. Appl. Phys. Commun. 1987, 6, 299.

(26) Armand, M.; Gorecki, W. Andreani Int. Symp. Polym. Electrolytes 1990, 31.

(27) Monroe, C.; Newman, J. J. Electrochem. Soc. 2005, 152, A396. 